\title{
Rhinolithiasis: A case report of a large rhinolith featuring the presence of granulation tissue.
}

\author{
Dr. Sujeet .Kumar ${ }^{1}$ Dr. Ramanuj. Singh ${ }^{2}$ Dr Subrata Nag ${ }^{3}$ \\ Assistant Professor, Dept, of ENT ${ }^{l}$,Assistant professor,Dept. of Anatomy ${ }^{2}$ Prof,Dept of Anesthesia ${ }^{3}$ \\ Narayan Medical College \& Hospital, Jamuhar, Sasaram, Bihar
}

\begin{abstract}
Rhinoliths rare nasal masses and related with the presence of foreign bodies. If undetected for a long time, they may grow large enough to cause symptoms of nasal obstruction, mimicking sinusitis . A case of a 35 year old woman having a 6 years old history of nasal obstruction, which was clinically and radiologically to be rhinolith featuring the presence of granulation tissue, is being reported.The mass was bulky and displaced posterioly into the nasopharynx and removed trans-orally with simultaneous resection of granulation tissue and application of $10 \%$ tricyclic acetic acid.
\end{abstract}

Key words: Rhinolithiasis ,nasal obstruction, granulation tissue ,tricyclic acetic acid

\section{Introduction}

The first recorded case of rhinolithiasis was reported by Barthdin in 1654.Rhinoliths are uncommon foreign bodies of the nose, which may be encountered accidentally during the course of a routine examination ${ }^{1}$. Their formation is caused by in situ calcification of intranasal endogenous or exogenous foreign material ${ }^{2}$.Rhinolithiasis is often an asymptomatic condition, which may remain undetected for many years ${ }^{3}$, until the rhinolith grow large enough to cause symptoms of nasal obstruction, discharge epistaxis ,erosion of the nasal septum and the medial wall of the maxillary sinus,leading the unwary physician to the erroneous diagnosis of rhinitis or unresolved sinus infection ${ }^{4}$.Therefore, in cases of permanent nasal obstruction, rhinolithasis must always be suspected in order to exclude malignancies or other pathological entities. The most common site of rhinolith is in the middle of the lower nasal duct and the majority of cases are unilateral. ${ }^{5}$ The case presented in this study concerns the existence of a large rhinolith to coexist with granulation tissue.

\section{Case Report}

A 35- year old female presented to the ENT department of NARAYAN MEDICAL COLLEGE \&HOSPITAL SASARAM, with a history of chronic nasal obstruction. The patient admitted frequent episode $\mathrm{s}$ of purulent rhinorrhea ,epistaxis ,intermittent pain in nose\&pain radiate to left eye ball and headache.On Anterior Rhinoscopic examination the left nasal cavity appeared completely obstructed because of the presence of a hard,blackish,irregular mass with granulation tissue \&foul-smelling pus around it.The mass which feels stony hard on Probing test.On Posterior rhinoscopic examination blackish irregular large mass seen in naspharyngeal region. X-ray PNS view revealed a calcified, radio -opaque shadow on the left side of the nose,accompanied by opacity of both maxillary antra(Figure 1). The rhinolith was bulky so first ,displaced posteriorly into the nasopharynx and removed trans-orally with simultaneously resection of granulaton tissue and removal of pus and application of $10 \%$ tricyclic acetic acid over remnant part of granulation tissue. The rhinolith was displaced posteriorly with help of freers elevator,retraction of both side of soft palate with help of Hajecks retactor ,opening of mouth and tongue depression with help of White-head mouth gag with tongue depressor and rhinolith was removed en masse ,through the trasorally,under general anesthesia wth the help of Luc's forceps(Figure 2).Specimens of granulations around the mass were removed and biopsed revealing chronic granulation tissue. Thepatient reported immediate relief of her symptoms after recovery from general anesthesia and has not had recurrence of nasal congestion or epistaxis during the next six months. 


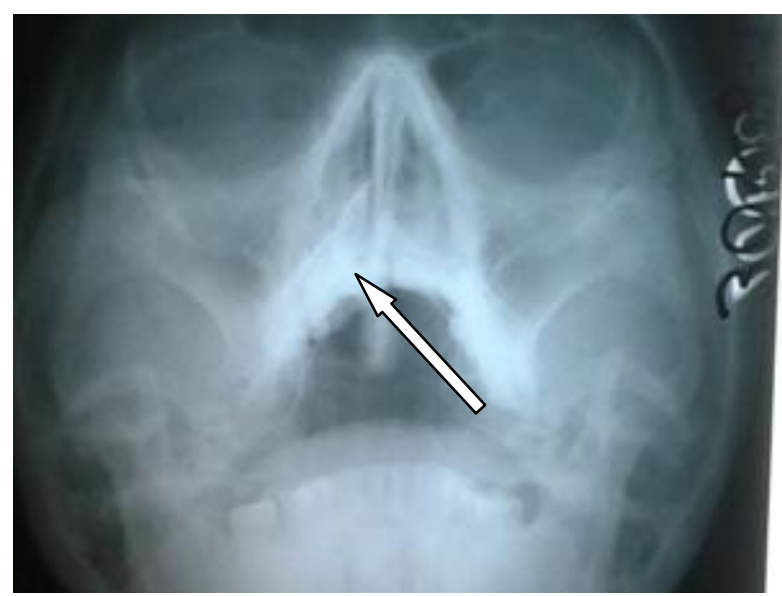

Figure 1 X-ray revealed an irregular calcified density in left nasal cavity,accompanied by opacity of both maxillary antra.

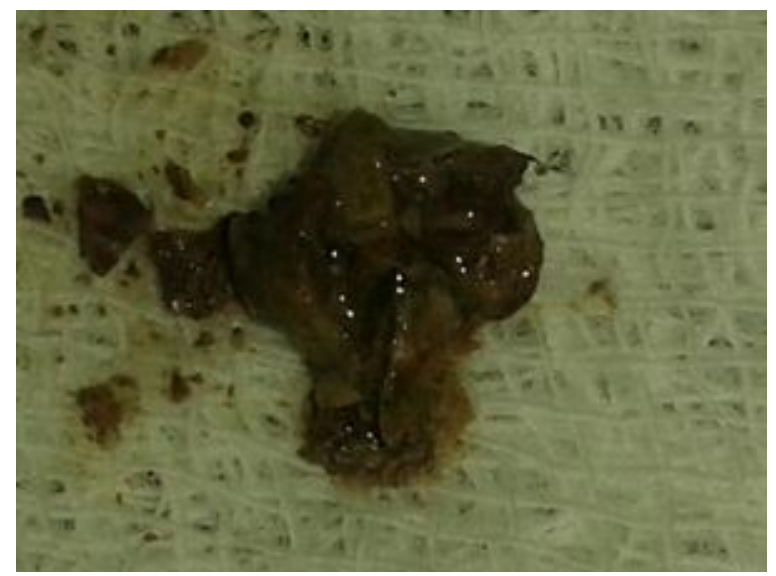

fig -2 Rhinolith after their trans-oral removal

\section{Discussion}

Rhinolith is thought to be formed by the gradual accretion of calcium and mineral salts around an intranasal nidus, which may be either endogenous or exogenous in origin.Exogenous calculi appear to be more common and these are usually beads, buttons fruit seeds, wood fragments sand, pieces of paper, fragments of bone and retained nasal packing.It seems that these had been introduced many years before probably during childhood and after having being chemically infected, are subsequently incrusted with calcified tissus. ${ }^{3}$ Endogenous calculi include dried nasal secretions,blood clots, epithelial debris, and sequestra ${ }^{10}$. The most extensive study is of Poloson et al in 1943 in which 495 cases are reviewed.In this study there is a predominance of female in sex, while ageof onset ranges from 6 month to 86 year. As the size of rhinoliths ncreases very slowly and they are relatively inert, they are initially symptomless and cause minor symptoms. ${ }^{3}$ If they become enough, they cause symptoms of nasal obstruction or chronic nasal discharge. Occasionally patients may complain of symptoms associated with local tissue distortion or destruction such as facial pain, swelling or epistaxis. These symptoms often raise the suspicion of a malignancy.Several complications have been occasionally described such as septal deviation ${ }^{11}$, septal perforation ${ }^{4}$, bony destruction and expansion of the calculus to involve the maxillary sinus ${ }^{12,2}$, oroantral and oronasal fistula ${ }^{13,14}$.A single case of meningitis has also been described ${ }^{15}$.

In most cases removal of the rhinolith is easy, through the anterior nares, either en masse or in pieces ${ }^{6}$.If they are bulky, they may be displaced posteriorly into the nasopharynx and removed trans-orally ${ }^{7}$. Extremely large and impacted calculi however may require a Caldwell -Luc approach ${ }^{8}$ or rare occasions, a lateral rhinotomy incision ${ }^{9}$.Endoscopic approaches can be performed in cases of small rhinoliths without complications ${ }^{6}$.An alternative method of removal is lithotripsyIn our patients the interesting fact is that the reactive tissue around the rhinolith appeared to be an granulation tissue as revealed by the first histopathological report.the finding could be verified by the final histopathological report. Removal en masse removal through trans-orally with simultaneously resection of granulation tissue and application of $10 \%$ tricyclic acetic acid for prevention or recurrence of granulation tissue. 


\section{Conclusion}

Although rhinoliths are quite uncommon,it is quite probable that an otolaryngologist will occasionally be confronted with such cases during his practice. Since clinical and radiological findings may be similar to other benign or malignant nasal lesions, knowledge of this clinical entity and a high degree of suspicion are necessary in order to accurately diagnosis and treat this condition.

\section{References}

[1]. Varley EWB(1964) Rhnolith:an incidental finding.Br J Oral Surg 2: 40-43.

[2]. Davis O, Wolff A (1985) Rhinolithiasis and maxillary antrolithiasis. Ear ,Nose and Throat J 64:421-426.

[3]. Carder HM, Hiel JJ (1966) Asymptomatic rhinolith: a brief review of the literature and case report. Laryngoscope 76:524-530.

[4]. Flood TR(1988) Rhinolith: an unusual cause of palatal perforation. Br J Oral maxillofacial Surg 26:486-490.

[5]. Appleton SS. Kimbrough RE, Engstrom HIM (1988) Rhinolithiasis : a review. Oral Surg 65:693-698.

[6]. Schwartz HC(1979) Rhinolithiasis :a disorder not to be approached transorally.J Am Dent Assoc 98:228.

[7]. Marano PD, Smart EA,Kolodny SC (1970) Rhinolith simulating osseous lesion:report of case.J Oral Surg 28 :615-616

[8]. Dutta A(1973) Rhinolith.J Oral 31:876-877.

[9]. Perrone JA (!968) An unusual rhinolith. Laryngoscope 78:279-280.

[10]. Harbin W, Weber AL (1979) Rhinoliths ann otol 88:578-579.

[11]. Deyasi S (1968) Rhinolith. J Laryngol otol 82:481-484.

[12]. Eliachar I, Schalit M (1970) Rhinolithiasis: report of eight cases. Arch Otolaryngol 91:88-90.

[13]. Gill RS,Lal M(1977) Perforation of the hard palate by a rhinolith and its repair .J Laryngol Otol 91:85-89

[14]. Wickham MH,Barton RPE(1988) Nasal regurgitation as the presenting symptom of rhinolithiasis.J Laryngol Otol 102:59-61

[15]. Polson JA (1943) On rhinoliths J Laryngol Otol 58:79-116.

[16]. Mink A,Gati I,Szekely J.Nasolith removal with ultrasound lithotripsy. HNO,1991;39:116-117. 